\title{
Neurological patients at risk of malnutrition are not always identified by the Malnutrition Universal Screening Tool
}

\author{
S. Yates ${ }^{1}$, A. H. M. Bonney ${ }^{1}$, K. Green ${ }^{1}$ and E. Harding ${ }^{2}$ \\ ${ }^{1}$ Department of Nutrition and Dietetics, The National Hospital for Neurology and Neurosurgery, Queen Square, London \\ WC1N 3BG, UK and ${ }^{2}$ Nutritional Sciences Division, King's College London, Stamford Street, London SE1 9NH, UK
}

Weight loss and malnutrition are well-known consequences of neurological conditions, often precipitated by dysphagia, hypercatabo$\operatorname{lism}^{(1)}$, or the disease progression itself ${ }^{(2,3)}$. At the National Hospital for Neurology and Neurosurgery, the Malnutrition Universal Screening Tool (MUST) is used to identify those who are at risk of malnutrition, and is completed on all inpatients on admission to the ward setting. In order to assess whether MUST is identifying all patients at risk of malnutrition in this predominantly neurological setting, a retrospective analysis of all patients seen over a 4-week period by the dietitians was completed to compare MUST score to reason for referral.

All patients seen by the dietitian between April and May 2009 were assessed. MUST score on admission was taken from the dietetic records, as well as weight, height, BMI, \% weight loss and reason for referral to dietitian.

In total, 52 patients were seen by the dietitians. Of these, 24 were deemed by MUST to be at a high risk of malnutrition, 5 at medium risk and 23 at low risk (see table). Of the 23 patients deemed at low risk, 5 were dysphagic and required a texture-modified diet, putting them at greater risk of malnutrition than identified by MUST. Three patients were provided with oral nutritional support as they were deemed to be at greater risk due to their diagnosis.

\begin{tabular}{ll}
\hline Malnutrition risk as deemed by MUST & \multicolumn{1}{c}{ Reason for dietetic intervention } \\
\hline High (MUST score $>2)$ & Oral nutritional support \\
& Requiring enteral nutritional support exclusively \\
Medium (MUST score of 1) & Requiring a texture-modified diet* \\
& Oral nutritional support \\
Low (MUST score of 0$)$ & Requiring a texture-modified diet \\
& Requiring enteral nutrition exclusively \\
& Requiring a texture-modified diet** \\
& Followed up after coming off enteral nutritional support \\
& Oral nutritional support \\
\hline
\end{tabular}

*all of whom were having additional partial enteral feeding.

**4 of whom were having additional partial enteral feeding.

These results suggest that although MUST screening is able to identify those already malnourished and some patients at risk of malnutrition, it cannot identify patients with specific symptoms such as dysphagia which can be detrimental to nutritional status, nor can it identify patients with hypercatabolic conditions/injuries such as motor neurone disease and some cerebral bleeds until weight loss has already occurred.

We therefore suggest that in the neurosciences setting, additional criteria such as dysphagia, the need for assistance, level of consciousness, prognosis and extent of injury should be considered when screening for malnutrition and identifying which patients require dietetic intervention.

1. Denes Z (2004) Disabil Rehab 26, 1163-1165

2. Heffernan C, Jenkinson C, Holmes T et al. (2004) Amyotroph Lateral Scler 5, 72-83.

3. Bachmann CG \& Trenkwalder C (2006) Mov Disord 21, 1824-1830. 